\title{
Quantification of Thin Specimens in a Scanning Transmission Electron Microscope at Low Accelerating Voltage using the f-ratio Method
}

\author{
Nicolas Brodusch ${ }^{1}$, Hendrix Demers $^{1}$ and Raynald Gauvin ${ }^{1}$ \\ ${ }^{1 .}$ Department of Mining and Materials Engineering, McGill University, Montreal, Quebec, Canada.
}

Since the last two decades, low voltage $\left(\mathrm{V}_{0} \leq 30 \mathrm{kV}\right)$ scanning transmission electron microscopy has gained interest due to its ability in increasing local contrast and reducing beam damage to beam sensitive materials compared to the more conventional high voltage operation of these microscopes. In a freestanding film, the relation between the intensity of the emitted x-rays and the true composition is highly dependent on the thickness of the film (Figure 1a). Furthermore, at low accelerating voltages absorption, fluorescence and electron channeling cannot be neglected. Due to this latter effect, the standards necessary for the quantification must have the same orientation as the analyzed specimen.

The method most commonly used to determine the concentrations of elements in a thin specimen is based on k-factors and is known as the Cliff-Lorimer method [1]. In this method, the absorption and fluorescence terms are neglected and the ratio of concentrations of elements $\mathrm{A}$ and $\mathrm{B}$ is related to the ratio of $\mathrm{x}$-ray intensities by the $\mathrm{k}_{\mathrm{AB}}$ factor $\left(\mathrm{C}_{A} / \mathrm{C}_{B}=\mathrm{k}_{\mathrm{AB}} \times \mathrm{I}_{\mathrm{A}} / \mathrm{I}_{B}\right)$. This factor can be determined either by using binary thin foils of known composition or by estimating them by calculations. This method is rapid and convenient if the database of $\mathrm{k}$-factors is available. To facilitate the determination of these factors, the $\zeta$ factor method [2] has been proposed where the concentration of an element is related to the x-ray intensity by the $\zeta$-factor which can be determined using single element thin foils of known thickness. This advantage makes this method easier to apply but still requires the tedious production of thin specimens and the knowledge of the beam current.

An alternative method for x-ray microanalysis in the electron microscope is the f-ratio method [3]. The fratio of each element is calculated by normalizing the intensity of this element over the sum of intensities from all elements present. A calibration curve is then necessary to link the f-ratios to the weight fractions and can be obtained using Monte Carlo simulations accounting for absorption and fluorescence. Results obtained on thin specimens of a Ti-6Al-4V alloy at $20 \mathrm{kV}$ in STEM mode are presented below. The net intensities (dashed lines) and the f-ratios (full lines) obtained by Monte Carlo simulations with the MC Xray software [4] were normalized to the bulk f-ratios and intensities, and are plotted in Figure 1a for Al, $\mathrm{Ti}, \mathrm{V}$, and $\mathrm{Fe}$ as a function of specimen thickness. Its impact on the f-ratios is dramatically reduced compared to the net intensities. In addition, as exemplified in Figure 1b-c, the f-ratio normalization was effective to cancel the effect of electron channelling contrast on x-ray emission and thus permitted to use Monte Carlo simulations to compute the calibration curve without accounting for the crystallographic orientation of the specimen. An example of such calibration curves is shown in Figure $2 \mathrm{a}$. A preliminary quantitative set of images in weight fractions obtained with this method is shown in Figure $2 \mathrm{~b}$-e for $\mathrm{Al}$, $\mathrm{Ti}, \mathrm{V}$, and Fe respectively.

\section{References:}

[1] Cliff, G. and G.W. Lorimer, Journal of Microscopy 103 (1975), p. 203.

[2] Watanabe, M. and D.B. Williams, J Microsc 221 (2006), p. 89.

[3] Horny, P., et al., Microscopy and Microanalysis 16 (2010), p. 821. 
[4] Gauvin, R. and P. Michaud, Microscopy and Microanalysis 15 (2009), p. 488.

a

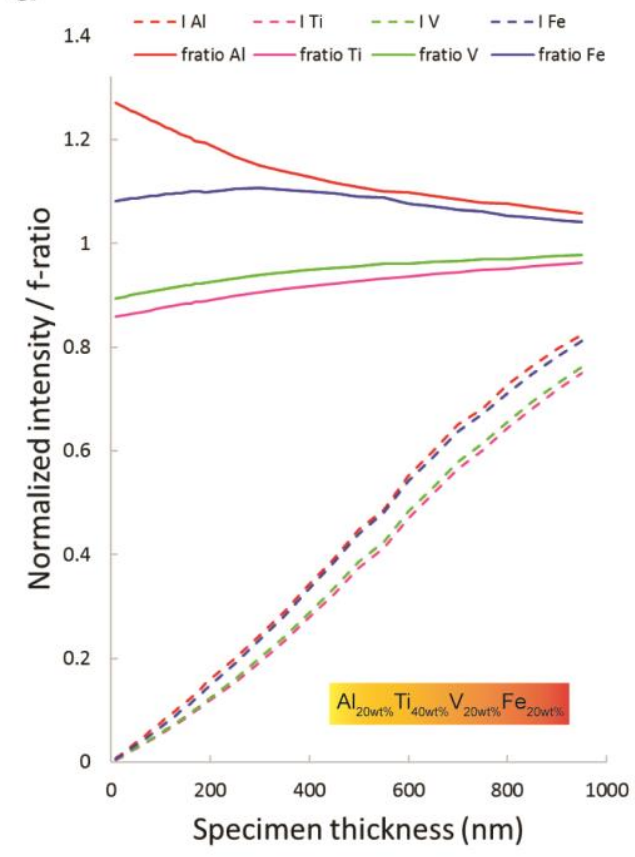

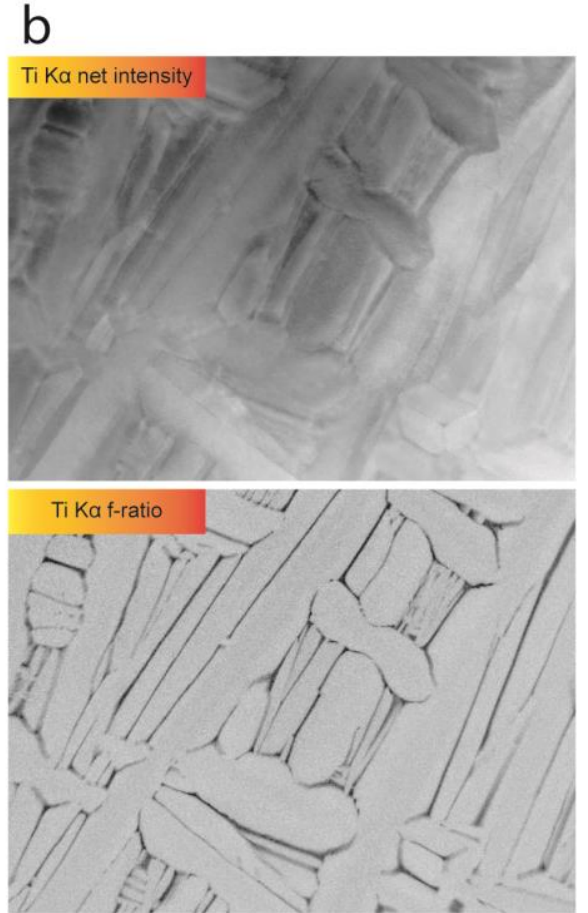

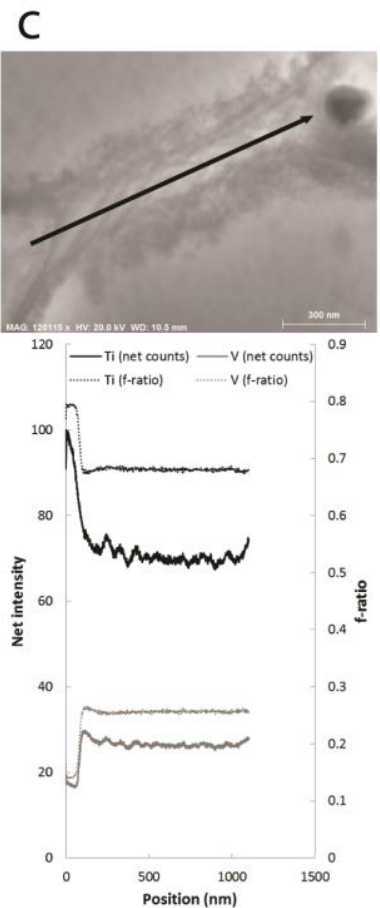

Figure 1. (a) Comparison between the emitted net $x$-ray intensities and the f-ratios calculated from the same intensities for a hypothetic Ti-20Al-20V-20Fe compound. The net intensities were obtained using the MC-X-Ray Monte Carlo simulation software [4] and the intensities and f-ratios were normalized with those obtained from the bulk for comparison purposes. (b) Ti Ka net intensity and f-ratio maps obtained with an annular SDD detector showing the effectiveness of the f-ratio method to cancel the electron channelling effect on $\mathrm{x}$-ray emission. (c) Line profile along a grain of $\beta$-phase showing nano-twinning with net intensities and f-ratio.

a

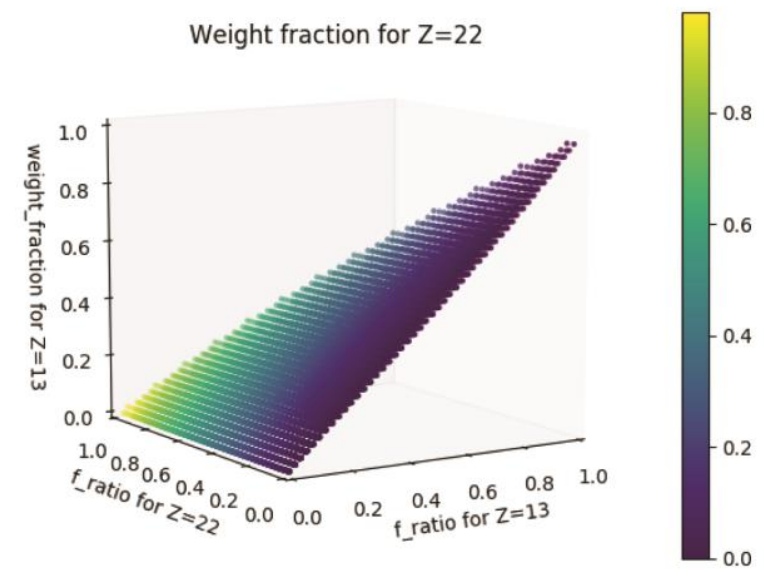

b

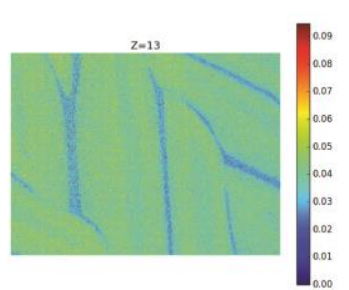

d

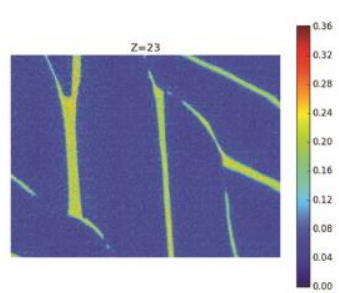

C

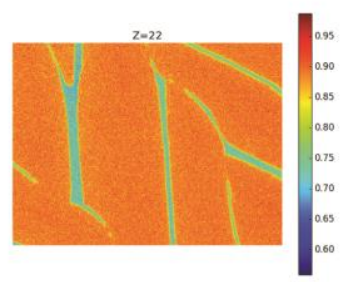

e

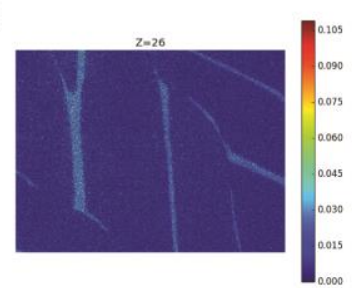

Figure 2. (a) Example of calibration curve linking the f-ratios to the weight fractions obtained via Monte Carlo simulations with the MC X-ray software [4]. (b-e) Quantitative weight fraction maps for Al, Ti, V, and Fe, respectively. 